\title{
Comparison of papanicolaou smear and human papillomavirus (HPV) test as cervical screening tools: can we rely on HPV test alone as a screening method? An 11-year retrospective experience at a single institution
}

\author{
Myunghee Kang, Seung Yeon Ha, Hyun Yee Cho, Dong Hae Chung, Na Rae Kim, \\ Jungsuk An, Sangho Lee, Jae Yeon Seok, Juhyeon Jeong \\ Department of Pathology, Gill Medical Center, Gachon University College of Medicine, Incheon, Korea
}

\begin{abstract}
Background: The decrease in incidence of cervical dysplasia and carcinoma has not been as dramatic as expected with the development of improved research tools and test methods. The human papillomavirus (HPV) test alone has been suggested for screening in some countries. The National Cancer Screening Project in Korea has applied Papanicolaou smears (Pap smears) as the screening method for cervical dysplasia and carcinoma. We evaluated the value of Pap smear and HPV testing as diagnostic screening tools in a single institution. Methods: Patients co-tested with HPV test and Pap smear simultaneously or within one month of each other were included in this study. Patients with only punch biopsy results were excluded because of sampling errors. A total of 999 cases were included, and the collected reports encompassed results of smear cytology, HPV subtypes, and histologic examinations. Results: Sensitivity and specificity of detecting high-grade squamous intraepithelial lesion (HSIL) and squamous cell carcinoma (SCC) were higher for Pap smears than for HPV tests (sensitivity, 97.14\%; specificity, 85.58\% for Pap smears; sensitivity, 88.32\%; specificity, 54.92\% for HPV tests). HPV tests and Pap smears did not differ greatly in detection of low-grade squamous intraepithelial lesion $(85.35 \%$ for HPV test, $80.31 \%$ for Pap smears). When atypical glandular cells were noted on Pap smears, the likelihood for histologic diagnosis of adenocarcinoma following Pap smear was higher than that of high-risk HPV test results (18.8 and 1.53, respectively). Conclusions: Pap smears were more useful than HPV tests in the diagnosis of HSIL, SCC, and glandular lesions.
\end{abstract}

Key Words: Uterine cervical neoplasms; Papanicolaou test; Human papillomavirus DNA tests; Early detection of cancer; Sensitivity

Received: August 13, 2019 Revised: November 28, 2019 Accepted: November 28, 2019

Corresponding Author: Seung Yeon Ha, MD, Department of Pathology, Gachon University Gil Medical Center, 21 Namdong-daero 774beon-gil, Namdong-gu, Incheon 21565, Korea Tel: +82-32-460-3078, Fax: +82-32-460-2394, E-mail: syha@gilhospital.com

Uterine cervical cancer is a well-understood malignancy. Information about the carcinogenesis, diagnosis, and vaccine prevention of cervical cancer is now available [1]. However, cervical cancer remains the third most common cause of death among females worldwide [2]. The incidence and mortality rates are diverse and are influenced by screening tests and public health infrastructure [2]. The incidence and mortality rates in Eastern Africa are about ten times higher than in Western Asia [2]. However, the incidence of cervical dysplasia and carcinoma have not decreased dramatically in areas with well-established public health programs. In developed countries, the estimated number of new cases has not decreased in four years, while the estimated number of deaths has increased from 33,500 cases in GLOBOCAN 2008 to 34,700 cases in GLOBOCAN 2012 [2,3].
For detection and treatment in the early stages, nationwide screening tests are needed. The Papanicolaou smear (Pap smears) was adopted as a screening test in 1999 for the National Cancer Screening Project in Korea. The Pap smear is performed biyearly in females over the age of thirty [4]. However, the use of human papillomavirus (HPV) DNA test as a screening method has been considered more helpful for protection against invasive cervical carcinomas in some studies [5]. The HPV DNA test has also been suggested as a screening method in some countries [6-8]. In the United States, some institutions proposed co-testing with Pap smears and HPV as a screening method [9]. In this study, we evaluated the value of Pap smears and HPV testing at a single institution. 


\section{MATERIALS AND METHODS}

\section{Case selection}

The medical records of 204,588 Pap smear cases at Gil Medical Center during the 11-year period from May 2006 to September 2017 were collected and retrospectively studied. Among these, 15,762 cases were HPV/Pap smear co-testing. Patients underwent HPV testing and Pap smears at the same time or within one month of each other. A total of 2,491 cases additionally underwent histologic evaluation. Patients with only punch biopsy results were excluded because of sampling errors. Inclusion criteria of the histologic results were reports of biopsies performed within 3 months of Pap smears and HPV testing. A total of 999 cases were included to include the results of smear cytology, HPV subtypes, and histologic examinations. Information on patient age, Pap smear results, HPV subtypes, surgical procedures, and pathologic results were collected.

\section{Pap smear evaluation}

Both conventional smear and liquid base preparations (Thin Prep Pap test, Cytyc Corporation, Boxborough, MA, USA) were included. Each case was reviewed by one cytotechnologist and one pathologist. The results were reported using the Bethesda system [10].

\section{HPV subtype testing}

During the 11-year period, the MyHPV chip kit (from March 2006 to February 2012, MyGene Co., Seoul, Korea), HPV DNA Chip (from March 2012 to February 2014, BioMedLab Inc., Chuncheon, Korea), and BMT HPV 9G DNA KIT (from March 2014 to September 2017, Biometrix Technology Inc., Chuncheon, Korea) were used to detect HPV. HPV tests were performed according to the manufacturer's instructions. Results were reported as positive or negative and as high-risk (HPV-16, $18,26,31,33,34,35,39,45,51,52,53,56,58,59,66,67$, 68, 69, 70, 73) or low-risk (HPV-3, 6, 10, 11, 27, 32, 40, 42, $43,44,54,55,57,61,62,72,74)$ subtypes. Cases showing HPV-negativity on the chip scanner but positivity under electrophoresis, i.e., not specified, were categorized as "other" after one repeat examination.

\section{Histologic evaluation}

When abnormal results were noted on cytology and/or HPV test, additional surgical procedures were performed after considering patient age, childbearing plans, and associated gynecologic diseases. If any patient had more than two pathologic results, the higher degree of abnormality was selected.

\section{Statistical analysis}

To evaluate the diagnostic accuracy, sensitivity, specificity, positive/negative likelihood ratio, and odds ratio were calculated along with their 95\% confidence intervals (CI) [11]. Cohen's kappa coefficient $(\kappa)$ was calculated using SPSS ver. 21.0 software (IBM Corp., Armonk, NY, USA). The $\kappa$ was interpreted categorically following the Fleiss guidelines of poor $(\kappa<0.4)$, fair $/ \operatorname{good}(\kappa=0.40$ to 0.75$)$, and perfect $(\kappa>0.75)$ [12].

\section{Ethics statement}

This study was reviewed and approved by the Institutional Review Board (IRB) of Gil Medical Center (No. GBIRB2019015). Formal written informed consent was not required due to a waiver from the appropriate IRB.

\section{RESULTS}

\section{Characteristics of cases}

A total of 999 cases fulfilled the inclusion criteria. Mean age was 46 years (range, 16 to 92 years). High-grade squamous intraepithelial lesion (HSIL) was the most common cytologic finding (473 cases, $46.7 \%$ ), followed by low-grade intraepithelial lesion (LSIL) (137 cases, 13.5\%) and negative for intraepithelial lesion or malignancy (NILM) (131 cases, 12.9\%). There were about five times more HPV positive cases than HPV-negative cases (830 cases, $83.1 \%)$. Cervical conization was performed more commonly than hysterectomy ( 723 cases, $72.4 \%$ ). Severe dysplasia/squamous carcinoma in situ (CIS) were the most common histologic findings (424 cases, 42.2\%). Clinicopathologic characteristics of patients are summarized in Table 1.

\section{Detection of squamous cell carcinomas and HSIL}

When HSIL cells were present on smear cytology, i.e., in cases of atypical squamous cells, cannot exclude HSIL (ASC-H), HSIL, and squamous cell carcinoma (SCC), the sensitivity was 97.14\% (95\% CI, 95.41 to 98.23) and specificity was $85.58 \%$ (95\% CI, 77.56 to 91.06). The sensitivity and specificity of highrisk HPV type test (sensitivity, 88.32\%; specificity, 54.92\%) were lower than that of Pap smears. These results are shown in Table 2. Sixteen cases showed benign cellular changes in the Pap smears $(16 / 654,2.45 \%)$, and 69 cases gave negative results on HPV tests $(69 / 654,10.55 \%)$. Seven cases gave negative results on both the Pap smears and the HPV tests (7/654, 1.07\%). 
Table 1. Clinicopathologic characteristics

\begin{tabular}{|c|c|}
\hline Characteristic & №. (\%) \\
\hline Age (yr), mean (range) & $46(16-92)$ \\
\hline \multicolumn{2}{|l|}{ Pap smear ${ }^{a}$} \\
\hline NILM & $131(12.9)$ \\
\hline ASCUS & $98(9.7)$ \\
\hline ASC-H & $70(6.9)$ \\
\hline LSIL & $137(13.5)$ \\
\hline HSIL & $473(46.7)$ \\
\hline SCC & $52(5.1)$ \\
\hline Atypical glandular cells & $24(2.4)$ \\
\hline AIS, adenocarcinoma & $26(2.6)$ \\
\hline Other malignant neoplasms & $3(0.3)$ \\
\hline \multicolumn{2}{|l|}{ HPV test } \\
\hline Negative & $169(16.9)$ \\
\hline Positive & $830(83.1)$ \\
\hline High-risk & $704(84.8)$ \\
\hline Low-risk & $14(1.7)$ \\
\hline Others & $112(13.5)$ \\
\hline \multicolumn{2}{|l|}{ Surgical procedures } \\
\hline Conization & $723(72.4)$ \\
\hline Hysterectomy & $276(27.6)$ \\
\hline \multicolumn{2}{|l|}{ Histologic results ${ }^{b}$} \\
\hline Normal & $150(14.9)$ \\
\hline Koilocytosis & $55(5.5)$ \\
\hline Mild dysplasia & $102(10.2)$ \\
\hline Moderate dysplasia & $116(11.5)$ \\
\hline Severe dysplasia, CIS & $424(42.2)$ \\
\hline SCC & $114(11.3)$ \\
\hline AIS & $10(1.0)$ \\
\hline Adenocarcinoma & $20(2.0)$ \\
\hline Adenosquamous cell carcinoma & $11(1.1)$ \\
\hline Others (malignant Mullerian mixed tumor) & $3(0.3)$ \\
\hline Total & 999 \\
\hline
\end{tabular}

NILM, negative for intraepithelial lesion or malignancy; ASCUS, atypical squamous cells of unknown significance; ASC-H, atypical squamous cells, cannot exclude HSIL; LSIL, low-grade squamous intraepithelial lesion; HSIL, high-grade squamous intraepithelial lesion; SCC, squamous cell carcinoma; AIS, adenocarcinoma in situ; CIS, carcinoma in situ.

aFifteen cases presented as two categorized results; ' ${ }^{\text {SSix }}$ cases presented as two categorized results.

\section{Detection of LSIL, including koilocytosis}

When atypical squamous cells of undetermined significance (ASCUS) or LSIL were identified in Pap smears, the sensitivity for LSIL detection was $80.31 \%$ (95\% CI, 72.55 to 86.30 ) and the specificity was $68.46 \%$ (95\% CI, 60.04 to 75.82 ). High and low-risk HPV tests (83.35\%; 95\% CI, 78.98 to 90.04) had higher sensitivity for detection of LSIL lesions than Pap smears; however, the $\mathrm{CI}$ overlapped. These results are shown in Table 2. Twenty-five cases had negative results on Pap smears (25/157, $15.92 \%)$, and 23 cases showed non-HPV infection (23/157, $14.65 \%)$. Four cases were negative by both Pap smear and HPV test $(4 / 157,2.55 \%)$.

\section{Detection of glandular lesions}

For detection of adenocarcinoma in situ (AIS) or adenocarcinoma, Pap smears were an excellent diagnostic tool. The sensitivity of the Pap smear was 100\% (95\% CI, 86.68 to 100) and specificity was $94.68 \%$ (95\% CI, 88.15 to 97.71 ). The sensitivity of high-risk type HPV test was $68.97 \%$ (95\% CI, 50.77 to 82.72 ) and specificity was $54.92 \%$ (95\% CI, 46.07 to 63.46). Nine cases were negative for $\operatorname{HPV}(9 / 30,30 \%)$. No cases were normal on Pap smears. Among nine HPV-negative cases, four revealed adenocarcinomas of endometrial origin and five were endocervical adenocarcinomas or AIS. One endocervical AIS case was accompanied by CIS.

\section{False positivity of Pap smears and HPV tests}

A total of 150 cases showed normal histologic results. Among them, 22 cases were diagnosed as abnormal by Pap smears (22/150, 14.67\%), and high-risk HPV was detected in 44 cases (44/150, 29.33\%). LSIL was the most common diagnosis from Pap smears (16 cases), followed by HSIL (three cases), atypical glandular cells (two cases), and ASC-H (one case). Of 999 total cases in this study, 16 cases had positive results on Pap smears and high-risk HPV but negative histologic results (16/999, $1.6 \%)$.

\section{Comparison of discrepancies between Pap smears and HPV test}

Among 999 cases, 180 showed discrepancies between cytology and HPV tests. The proportion of positivity on Pap smears was higher than that in HPV tests $(86.89 \%$ and $83.08 \%$, respectively), and $\kappa$ was 0.30 (95\% CI, 0.22 to 0.37 ). The $\kappa$ between Pap smears and histologic results was 0.57 (95\% CI, 0.50 to 0.65$)$. The $\kappa$ between HPV test and histologic results was 0.31 (95\% CI, 0.23 to 0.39). These results are shown in Table 3 .

\section{DISCUSSION}

The present study was performed at a single institution in Korea to evaluate the value of Pap smears and HPV DNA tests for diagnosing cervical neoplasms. Pap smears had higher sensitivity and specificity than HPV DNA tests for HSIL, SCC, and glandular lesions. The sensitivity of HPV tests for LSIL was higher than that of Pap smears; however, the difference was not significant because of overlapping CI. Odds ratios of Pap smears were higher than that of HPV tests for all histologic categories. This study showed higher sensitivity and specificity for Pap smears compared with previously reported studies (sensitivity, 
Table 2. Sensitivity, specificity, positive LR, negative LR and OR with histologic confirmation

\begin{tabular}{|c|c|c|c|c|c|}
\hline Histologic results & Sensitivity & Specificity & Positive LR & Negative LR & OR \\
\hline \multicolumn{6}{|l|}{ SCC/HSIL } \\
\hline ASC-H/HSIL/SCC on Pap smear & $97.14(95.41-98.23)$ & $85.58(77.56-91.06)$ & $6.74(4.22-10.76)$ & $0.03(0.02-0.05)$ & $201.73(96.33-422.48)$ \\
\hline hrHPV+ & $88.32(85.48-90.67)$ & $54.92(46.07-63.46)$ & $1.96(1.61-2.39)$ & $0.21(0.16-0.28)$ & $9.22(5.96-14.25)$ \\
\hline \multicolumn{6}{|l|}{ LSIL } \\
\hline ASCUS/LSIL on Pap smear & $80.31(72.55-86.30)$ & $68.46(60.04-75.82)$ & $2.55(1.95-3.33)$ & $0.29(0.20-0.42)$ & $8.86(4.99-15.71)$ \\
\hline $\mathrm{HPV}+^{\mathrm{a}}$ & $85.35(78.98-90.04)$ & $44.67(36.94-52.66)$ & $1.54(1.32-1.81)$ & $0.33(0.22-0.50)$ & $4.7(2.72-8.13)$ \\
\hline \multicolumn{6}{|l|}{ AIS, adenocarcinoma } \\
\hline Glandular cell abnormality on Pap smear & $100(86.68-100)$ & $94.68(88.15-97.71)$ & $18.8(8.01-44.11)$ & NA & NA \\
\hline hrHPV+ & $68.97(50.77-82.72)$ & $54.92(46.07-63.46)$ & $1.530(1.12-2.09)$ & $0.57(0.32-0.99)$ & $2.71(1.14-6.42)$ \\
\hline
\end{tabular}

Numbers in parentheses indicate $95 \%$ confidence interval.

LR, likelihood ratio; OR, odds ratio; SCC, squamous cell carcinoma; HSIL, high-grade squamous intraepithelial lesion; ASC-H, atypical squamous cells, cannot exclude HSIL; hr, high-risk; LSIL, low-grade intraepithelial lesion; ASCUS, atypical squamous cells of undetermined significance; Pap smear, Papanicolaou smear; HPV, human papillomavirus; AIS, adenocarcinoma in situ; NA, not available.

aHPV included low- and high-risk HPV types.

Table 3. Kappa coefficients of Pap smear, HPV and histology

\begin{tabular}{lcccc} 
& $\kappa$ & Standard error of $\kappa$ & $95 \%$ confidence interval & The strength of agreement $^{\mathrm{a}}$ \\
\hline $\begin{array}{l}\text { Pap smear } \\
\text { HPV }\end{array}$ & 0.30 & 0.04 & $0.22-0.37$ & Poor \\
$\begin{array}{l}\text { Pap smear } \\
\text { Histology }\end{array}$ & 0.57 & 0.04 & $0.50-0.65$ & Fair/good \\
$\begin{array}{l}\text { Pap smear } \\
\text { Histology }\end{array}$ & 0.31 & 0.04 & $0.23-0.39$ & Poor \\
$\begin{array}{l}\text { Pap smear and/or HPV } \\
\text { Histology }\end{array}$ & 0.42 & 0.04 & $0.33-0.50$ & Fair/good \\
\hline
\end{tabular}

Pap smear, Papanicolaou smear; HPV, human papillomavirus.

aThese categories were classified by Fleiss guidelines.

49\%-84\%; specificity, 45\%-84\%) [13-16]. Sensitivity and specificity of high-risk HPV tests were similar in previous studies (sensitivity, 74\%-96\%; specificity, 59\%-80\%) [13-16]. This is explained by the multiple review systems used in this study. Rules of the Clinical Laboratory Improvement Amendments (CLIA) 1988 mandated 10\% random rescreening process in cases of NILM category of the Bethesda system were applied. However, many slides were actually reviewed to train pathology residents or cytotechnologists. More than one pathologist, each with more than five years of experience, reviewed almost all cases.

Most cases in this study were from the period without HPV vaccination. Quadrivalent HPV vaccines were approved in Korea in 2006, and a nationwide vaccination program was adopted in 2016. Vaccine effects require more time to stop carcinogenesis [17]. In addition, the prevalence of LSIL or HSIL in this specific hospital was higher than that in most other institutions, as reported in nationwide screening results [18]. Between 2009 and 2014, the diagnostic rates of LSIL and HSIL were 1.32\% and $0.62 \%$, respectively, at our hospital. In the Korea nationwide survey, the diagnostic rates of LSIL and HSIL were $0.24 \%$ and $0.14 \%$, respectively [18]. There is a higher chance of encountering squamous intraepithelial lesions in the clinical field at tertiary referral hospitals. The thorough slide review might have led to the high specificity and sensitivity of Pap smears. Furthermore, the number of detectable HPV subtypes increased with changes in HPV test kits three times, from 24 subtypes in 2006 to 38 subtypes in 2017.

The false-negative rates of HPV tests were higher than those of Pap smears for SCC, HSIL, and glandular lesions. In cases of negative HPV detection results and HSIL/SCC in surgical specimens, HPV DNA test samples were re-examined through formalin-fixed paraffin embedded blocks. In this study, $10.55 \%$ of HSIL/SCC cases (69/654) were not detected through HPV tests. Among these, 51 cases were diagnosed as ASC-H/HSIL/SCC $(51 / 69,73.91 \%)$ from Pap smears and 11 were diagnosed as LSIL/ASCUS $(11 / 69,15.94 \%)$. Only seven cases were NILM on Pap smears $(7 / 69,10.15 \%)$. This might have been due to a low viral load or HPV-negative squamous cell carcinoma [19-21]. It has been suggested that progression of cervical dysplasia does not require continuous HPV replication. Therefore, the degree of dysplasia does not correlate with HPV load. About $5 \%$ of SCCs are HPV-negative [21]. In the present study, HPV-negative SCCs constituted $9.65 \%$ (11/114) of SCCs. Among these, only one case showed normal cytology on Pap smear. When us- 
ing Pap smear to detect SCC, the false-negative rate was $0.88 \%$.

In this study, Pap smears proved more promising than HPV tests to detect glandular lesions. In addition, $16.6 \%$ of endocervical AIS or adenocarcinoma cases (5/30) were negative on HPV tests. These findings are in agreement with previous observations on the existence of HPV-negative endocervical adenocarcinoma (about 15\%) [22]. The Pap smears and histologic slides of five cases of HPV-negative endocervical AIS/adenocarcinoma were reviewed. Three cases were endometrioid type adenocarcinoma and one was gastric type adenocarcinoma [23]. Gastric type adenocarcinoma is an HPV-unrelated cancer, and endometrioid type adenocarcinoma is commonly associated with cervical endometriosis. We did not identify cervical endometriosis in these three cases. The relationship between endocervical adenocarcinoma, endometrioid adenocarcinoma, and HPV infection is not fully understood [22-24]. One case diagnosed as cervical AIS was accompanied by squamous CIS. The negative HPV test result may have been a false-negative. Among the nine cases of HPV-negative adenocarcinoma in this study, four cases revealed an endometrial origin. These cases presented with vaginal bleeding, and Pap smears and HPV tests were initially performed with colposcopic examination. Ultrasonography and endometrial biopsy or curettage usually follow in the routine clinical setting. Endometrial dilation and curettage are performed under anesthesia; however, Pap smears are easily performed. Similar to previously reported results, we found that Pap smears were useful in detection of glandular lesions [25].

Co-testing of Pap smears and HPV yielded higher sensitivity and specificity in detection of HSIL than one test alone in some previous studies [26-28]. In the present study, sensitivity (98.91\%; 95\% CI, 97.75 to 99.47 ) was higher but specificity (44.55\%; $95 \%$ CI, 35.60 to 53.86) was lower than with a single test alone. This may result from high false-positive rates in this study pool. Transient HPV infection was considered one reason for the high false-positive rate. About $90 \%$ of infections were eliminated within three years [29]. Additionally, some previously treated lesions showed atypical cells on Pap smears during the wound healing period, which may be diagnosed as ASCUS or LSIL on Pap smear. Among 20 cases of false positives on Pap smears and HPV tests, three cases were diagnosed as HSIL, and two cases demonstrated glandular cell abnormality on cytology. One case of HSIL presented with marked inflammatory cells and red blood cells on the smear; therefore, misinterpretation could have occurred due to artifact. In the two other cases of HSIL, the patient had previously undergone conization. The Pap smears and HPV tests in this study were follow-up examina- tions, and two patients underwent repeat conization. Specimens after a second conization were small and fragmented. In addition, they had ambiguous orientation and cautery artifact, resulting in questionable histologic results. One patient diagnosed with atypical glandular cells underwent hysterectomy to treat a large leiomyoma. No grossly abnormal lesions were noted in the cervix. Therefore, only two representative sections of cervix were submitted. If all cervical tissues had been submitted and reviewed by microscopy, atypical glandular lesion may have been diagnosed. The other case of glandular cell abnormality presented as adenocarcinoma cells on Pap smear. This patient underwent conization, and additional treatment was proposed. However, the patient refused treatment. Based on these cases, the false-positive rate might be due to misdiagnosis on Pap smear or under-diagnosis on histologic examination or clinical procedures.

In this study, we found that Pap smears are still more useful than HPV DNA tests in diagnosing HSIL, SCC, and glandular lesions. Pap smears are indicated for diagnosis of non-HPV related cervical adenocarcinomas or endometrial lesions. Additionally, Pap smears may provide information on the degree of squamous dysplasia, i.e., high grade versus low grade. There are some limitations because the present study did not consider disease progression or elimination of HPV infections.

\section{ORCID}

Myunghee Kang: https://orcid.org/0000-0003-4083-888X

Seung Yeon Ha: https://orcid.org/0000-0001-7071-1623

Hyun Yee Cho: https://orcid.org/0000-0003-3603-5750

Dong Hae Chung: https://orcid.org/0000-0002-4538-0989

Na Rae Kim: https://orcid.org/0000-0003-2793-6856

Jungsuk An: https://orcid.org/0000-0003-0312-2460

Sangho Lee: https://orcid.org/0000-0003-4636-7521

Jae Yeon Seok: https://orcid.org/0000-0002-9567-6796

Juhyeon Jeong: https://orcid.org/0000-0002-8665-0295

\section{Author Contributions}

Conceptualization: SYH.

Data curation: JJ.

Formal analysis: SL.

Investigation: JYS.

Methodology: DHC.

Project administration: NRK.

Supervision: SYH, HYC.

Validation: JA. 
Visualization: JYS.

Writing—original draft: MK, SYH.

Writing—review \& editing: MK, SYH.

\section{Conflicts of Interest}

S.Y.H., a contributing editor of the Journal of Pathology and Translational Medicine, was not involved in the editorial evaluation or decision to publish this article. All remaining authors have declared no conflicts of interest.

\section{Funding}

This research was supported by The Korean Society for Cytopathology Grant.

\section{REFERENCES}

1. Snijders PJ, Steenbergen RD, Heideman DA, Meijer CJ. HPV-mediated cervical carcinogenesis: concepts and clinical implications. J Pathol 2006; 208: 152-64.

2. Torre LA, Bray F, Siegel RL, Ferlay J, Lortet-Tieulent J, Jemal A. Global cancer statistics, 2012. CA Cancer J Clin 2015; 65: 87-108.

3. Jemal A, Bray F, Center MM, Ferlay J, Ward E, Forman D. Global cancer statistics. CA Cancer J Clin 2011; 61: 69-90.

4. Kim Y, Jun JK, Choi KS, Lee HY, Park EC. Overview of the National Cancer screening programme and the cancer screening status in Korea. Asian Pac J Cancer Prev 2011; 12: 725-30.

5. Polman NJ, Snijders PJ, Kenter GG, Berkhof J, Meijer CJ. HPVbased cervical screening: Rationale, expectations and future perspectives of the new Dutch screening programme. Prev Med 2019; 119: 108-17.

6. Sangrajrang S, Laowahutanont P, Wongsena M, et al. Comparative accuracy of Pap smear and HPV screening in Ubon Ratchathani in Thailand. Papillomavirus Res 2017; 3: 30-5.

7. Ronco G, Dillner J, Elfstrom KM, et al. Efficacy of HPV-based screening for prevention of invasive cervical cancer: follow-up of four European randomised controlled trials. Lancet 2014; 383: 52432.

8. Tota JE, Bentley J, Blake J, et al. Introduction of molecular HPV testing as the primary technology in cervical cancer screening: acting on evidence to change the current paradigm. Prev Med 2017; 98: 5-14.

9. US Preventive Services Task Force, Curry SJ, Krist AH, et al. Screening for cervical cancer: US Preventive Services Task Force recommendation statement. JAMA 2018; 320: 674-86.

10. Nayar R, Wilbur DC. The Bethesda system for reporting cervical cytology: definitions, criteria, and explanatory notes. 3rd ed. Cham:
Springer, 2015.

11. Brown LD, Cai TT, DasGupta A. Interval estimation for a binomial proportion. Stat Sci 2001; 16: 101-33.

12. Fleiss JL. Statistical methods for rates and proportions. 2nd ed. New York: John Wiley, 1981.

13. Tracht J, Wrenn A, Eltoum IE. Primary HPV testing verification: a retrospective ad-hoc analysis of screening algorithms on women doubly tested for cytology and HPV. Diagn Cytopathol 2017; 45: 580-6.

14. Costa S, Sideri M, Negri G, et al. The predictive value of human papillomavirus testing for the outcome of patients conservatively treated for stage IA squamous cell cervical carcinoma. J Clin Virol 2015; 70: 53-7.

15. McKenna M, McMenamin MM. Human papillomavirus testing in young women: clinical outcomes of human papillomavirus triage in a UK cervical screening program. Cancer Cytopathol 2014; 122: 702-10.

16. Pileggi C, Flotta D, Bianco A, Nobile CG, Pavia M. Is HPV DNA testing specificity comparable to that of cytological testing in primary cervical cancer screening? Results of a meta-analysis of randomized controlled trials. Int J Cancer 2014; 135: 166-77.

17. Garland SM, Kjaer SK, Muñoz N, et al. Impact and effectiveness of the quadrivalent human papillomavirus vaccine: a systematic review of 10 years of real-world experience. Clin Infect Dis 2016; 63: 519-27.

18. Shim SH, Kim H, Sohn IS, et al. Nationwide cervical cancer screening in Korea: data from the National Health Insurance Service Cancer Screening Program and National Cancer Screening Program, 2009-2014. J Gynecol Oncol 2017; 28: e63.

19. Sherman ME, Wang SS, Wheeler CM, et al. Determinants of human papillomavirus load among women with histological cervical intraepithelial neoplasia 3: dominant impact of surrounding lowgrade lesions. Cancer Epidemiol Biomarkers Prev 2003; 12: 1038-44.

20. Jastania R, Geddie WR, Chapman W, Boerner S. Characteristics of apparently false-negative digene hybrid capture 2 high-risk HPV DNA testing. Am J Clin Pathol 2006; 125: 223-8.

21. Cancer Genome Atlas Research Network, Albert Einstein College of Medicine; Analytical Biological Services, et al. Integrated genomic and molecular characterization of cervical cancer. Nature 2017; 543: 378-84.

22. Stolnicu S, Barsan I, Hoang L, et al. International Endocervical Adenocarcinoma Criteria and Classification (IECC): a new pathogenetic classification for invasive adenocarcinomas of the endocervix. Am J Surg Pathol 2018; 42: 214-26.

23. Hodgson A, Park KJ, Djordjevic B, et al. International endocervical adenocarcinoma criteria and classification: validation and interob- 
server reproducibility. Am J Surg Pathol 2019; 43: 75-83.

24. Kurman RJ, Carcangiu ML, Herrington CS, Young RH. WHO classification of tumours of female reproductive organs. 4th ed. Lyon: IARC Press, 2014.

25. Nasser H, AlAyyaf M, Atallah A, et al. Eleven-year review of data on Pap smears in Saudi Arabia: we need more focus on glandular abnormalities! Ann Saudi Med 2017; 37: 265-71.

26. Patanwala IY, Bauer HM, Miyamoto J, Park IU, Huchko MJ, SmithMcCune KK. A systematic review of randomized trials assessing human papillomavirus testing in cervical acancer screening. Am J Obstet Gynecol 2013; 208: 343-53.
27. Kitchener HC, Almonte M, Thomson C, et al. HPV testing in combination with liquid-based cytology in primary cervical screening (ARTISTIC): a randomised controlled trial. Lancet Oncol 2009; 10: 672-82.

28. Luyten A, Buttmann-Schweiger N, Luyten K, et al. Early detection of CIN3 and cervical cancer during long-term follow-up using $\mathrm{HPV} /$ Pap smear co-testing and risk-adapted follow-up in a locally organised screening programme. Int J Cancer 2014; 135: 1408-16.

29. Sasagawa T, Takagi H, Makinoda S. Immune responses against human papillomavirus (HPV) infection and evasion of host defense in cervical cancer. J Infect Chemother 2012; 18: 807-15. 